\title{
Relevance of pressure field accuracy for nonlinear Froude-Krylov force calculations for wave energy devices
}

\author{
Giuseppe Giorgi $^{1}$ (D) $\cdot$ John V. Ringwood ${ }^{1}$
}

Received: 7 September 2017 / Accepted: 18 December 2017

c) Springer International Publishing AG, part of Springer Nature 2017

\begin{abstract}
Due to their computational convenience, linear mathematical models for wave energy converters are usually employed. Including nonlinearities may improve the accuracy of the results, but often at the price of an additional computational and complexity burden, which can be justified only if nonlinearities are significant. One of the sources of nonlinearity in fluid-body interactions is the wave field itself. Different wave models exist, among which are linear Airy's theory, Wheeler's stretching approach, the nonlinear Rienecker-Fenton method, and higher order spectral methods, all of which achieve a different compromise of accuracy and complexity. The impact of the accuracy of such wave theories strongly depends on the specific device (operating principle, power production region or survivability mode), and installation site (water depth, occurrences of each sea state in the scatter diagram of the installation site). This paper evaluates the performance of different wave field representations, firstly in absolute terms and, secondly, in relation to the associated computation of nonlinear Froude-Krylov forces for different wave energy devices, in regular and irregular sea states. It is shown that Wheeler's stretching offers a good accuracy/complexity compromise for WECs operating in the power production region.
\end{abstract}

Keywords Nonlinear wave $\cdot$ Wheeler's stretching $\cdot$ Rienecker-Fenton wave $\cdot$ Higher order spectral method $\cdot$ Nonlinear Froude-Krylov force · Wave energy converters

\section{Introduction}

Due to their computational convenience, linear mathematical models for wave energy converters (WECs) are usually employed. Including nonlinearities may improve the accuracy of the results, but often at the price of an additional computational and complexity burden, which can be justified only if nonlinearities are significant.

Nonlinearities in fluid-body interactions depend on:

(i) The free surface elevation

(ii) The pressure field distribution

(iii) Geometric nonlinearities.

Giuseppe Giorgi

giuseppe.giorgi.2015@mumail.ie

John V. Ringwood

john.ringwood@nuim.ie

1 Centre for Ocean Energy Research, Maynooth University, Maynooth, Co.Kildare, Ireland
Points (i) and (ii) concern the modelling of the wave field, which is the energy source, while point (iii) concerns the computation of the hydrodynamic forces acting on the body, which are defined as the integral of the pressure acting on the wetted surface of the device. In particular, FroudeKrylov (FK) forces are the hydrodynamic components which describe the action of the undisturbed incident wave on a device; therefore, accurate modelling of FK forces is likely to be important for reliably simulating responses of wave energy devices. For example, Giorgi et al. (2016) show that geometric nonlinearities may be relevant for the FK force calculation for heaving point absorbers (HPAs), significantly affecting the response of the device. Indeed, FK forces are the predominant hydrodynamic component for WECs whose characteristic dimension is much smaller than the wavelength, implying relatively small diffraction and radiation effects. However, the notion of decomposition of the whole hydrodynamic force into FK, radiation, and diffraction components, as well as the spectral decomposition of the incident irregular wave train, inherently relies on the superposition principle. Such an assumption can be safely considered valid, as long as the sea state is not extreme. Therefore, the scope 
of application of nonlinear FK models covers only the power production region, while not extending to the survivability region (Penalba et al. 2017).

Mathematical models, in the power production region, are used for design, optimization studies, power production assessment, control, and array layout definition; therefore, in addition to an accuracy requirement, such models also need to be fast to compute. Including geometric nonlinearities for the computation of nonlinear FK forces implies an increase in complexity and computational time, which depends on the complexity of the geometry itself; for relatively simple geometries, such as axisymmetric heaving point absorbers, or oscillating wave surge converters (OWSCs), computationally-efficient algebraic solutions are available (Giorgi and Ringwood 2017c), while more complex geometries require alternative approaches (Gilloteaux 2007). Furthermore, while linear FK forces are computed with respect to the still water level, nonlinear FK force calculations require knowledge of the pressure distribution up to the actual free surface elevation. Therefore, the accuracy of the FK force model is directly related to the accuracy of the pressure field model. Likewise, the complexity of the pressure formulation has a significant influence on the computational effort required to compute nonlinear FK forces.

In the last decade, nonlinear FK models have gradually become more popular in the wave energy field (Gilloteaux 2007; Babarit et al. 2009; Merigaud et al. 2012; Tarrant 2015; Giorgi and Ringwood 2017c; Guerinel et al. 2017). However, the vast majority of such models just considers the pressure representation from first-order linear Airy's theory. Different degrees of accuracy (at different complexity costs) can be achieved in modelling the wave field. For the basic case of steady (regular) waves, the simplest approach is linear Airy's theory, followed by several nonlinear wave formulations, including Stokes' waves (2nd-4th order), cnoidal waves, and Rienecker-Fenton (RF) waves (Fenton 1990). Finally, corrections to linear theory, such as Wheeler's (1970) stretching method, can be used to improve the results of Airy's theory, at a negligible additional complexity cost. For irregular waves, higher order spectral (HOS) formulations are often used, since they can efficiently solve the nonlinear potential problem, as well as the nonlinear propagation problem (Ochi 2005). The accuracy of such wave theories depends mainly on the water depth, wave steepness, and wave height; therefore, the impact of such nonlinearity strongly depends on the specific device (position of the device with respect to the free surface and the sea bottom, power production region or survivability mode), and installation site (water depth, occurrence of each sea state in the scatter diagram of the installation site).

This paper discusses the impact that the choice of the wave field representation model has on the accuracy and complexity of the calculation of nonlinear FK forces for wave energy devices. Regular and irregular waves are considered, using Airy's theory, Wheeler's stretching, Rienecker-Fenton waves, and HOS methods. Initially, the pressure profile is evaluated and compared, from the free surface to the sea bottom, for a comprehensive set of wave conditions. Secondly, the effect of different pressure field representations on the calculation of nonlinear FK forces for WECs is discussed, considering HPAs and OWSCs as representative devices. While the accuracy of the pressure field representation has already been discussed in the literature [using Wheeler's stretching (Du et al. 2009; Clauss et al. 2009), RieneckerFenton (Fenton 1990), and HOS (Ducrozet et al. 2007)], to the best of the authors' knowledge, the present paper is the first study considering the impact of the pressure field accuracy on nonlinear FK force calculations for wave energy devices.

The remainder of the paper is organized as follows: Sect. 2 presents the wave models, based on potential theory. Sections 3 and 4 consider regular and irregular waves, respectively. The overall accuracy of the pressure profile representation is discussed in Sects. 3.1 and 4.1, while the effect of the pressure representation on the calculation of nonlinear FK forces is discussed in Sects. 3.2 and 4.2. Some final remarks and conclusions are presented in Sect. 5.

\section{Wave theories}

The wave theories discussed herein are based on the assumption of a homogeneous, ideal, incompressible fluid, with irrotational motion (Newman 1977). Two-dimensional waves are considered in the $(x, z)$ coordinate system, where $x$ is the direction of propagation of the wave, and $z$ is the vertical axis, positive upwards, with the origin at the still water level (SWL), which is at a distance $d$ from the sea bed. Assuming the flow is irrotational, a velocity potential $\varphi$ can be defined, such that:

$\mathbf{u}=\nabla \varphi$,

where $\mathbf{u}$ is the velocity vector. Since the motion is assumed incompressible, $\varphi$ satisfies Laplace's equation throughout the fluid:

$\nabla \times \mathbf{u}=\nabla^{2} \varphi=0$.

The kinematic boundary conditions are satisfied on the flat sea bottom (3a), and on the free surface $\eta(3 b)$ :

$$
\begin{aligned}
& \frac{\partial \varphi}{\partial z}=0, \quad \text { at } z=-d, \\
& \frac{\partial \varphi}{\partial z}=\frac{\partial \eta}{\partial t}+\frac{\partial \varphi}{\partial x} \frac{\partial \eta}{\partial x}, \quad \text { at } z=\eta
\end{aligned}
$$


Finally, the dynamic boundary condition (Bernoulli's equation) is verified on the free surface:

$\frac{\partial \varphi}{\partial t}+\frac{p}{\rho}+g z+\frac{|\nabla \varphi|^{2}}{2}=0, \quad$ at $z=\eta$,

where $\rho$ is the water density, $g$ the acceleration of gravity, and $p$ the total pressure.

The resolution of such a potential problem is different, depending on the use of regular or irregular waves. In Sect. 2.1, different regular wave theories are discussed, while Sect. 2.2 is concerned with irregular sea state representations.

\subsection{Regular waves}

A general analytical solution to the wave velocity potential problem, described by Eqs. (1)-(4), does not exist. In particular, some further assumption are needed to solve the free surface boundary conditions (3b) and (4): Airy's wave theory linearizes such boundary conditions onto the SWL, while Stokes' wave theory, assuming infinite water depth (with respect to the wave length $\lambda$ ), uses a Fourier series of (3b) and (4), performing a perturbation expansion in terms of a deviation parameter, which increases with the wave steepness. On the other hand, cnoidal wave theory solves the potential problem, assuming that the wave length is much longer than the water depth.

The regions of validity of such wave theories are shown in Fig. 1. Note that there are different orders of Stokes' theory, where first order corresponds to linear Airy's theory.

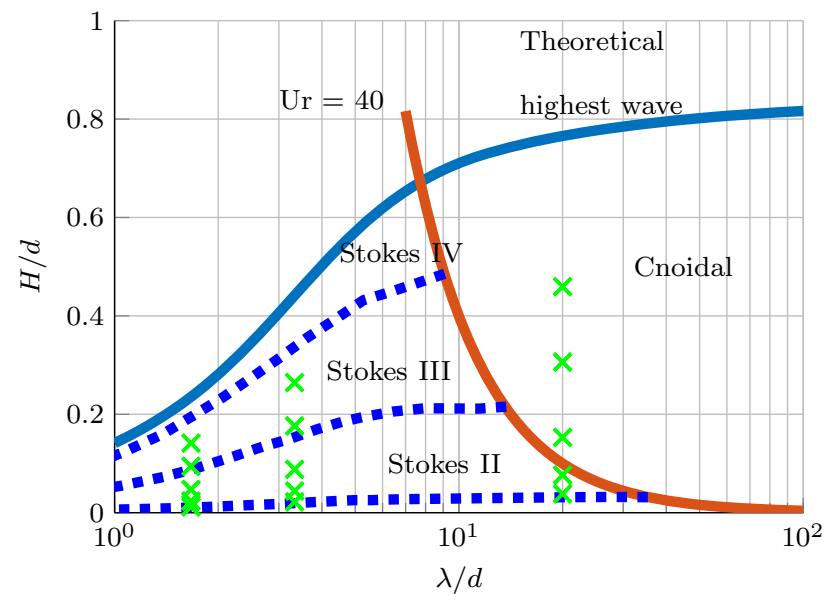

Fig. 1 Regions of validity of wave theories, based on (Le Méhauté 1976), for different wave lengths $\lambda$ and wave heights $H$, both normalized with respect to the water depth $d$. The boundary between cnoidal and Stokes wave theories is given by an Ursell number (Ur) of 40 (Hedges 1995). The thresholds between consecutive orders of the Stokes wave (dashed lines) are qualitative. The theoretical highest wave possible is based on Williams (1981). The markers on the graph refer to the wave conditions studied in Sect. 3.2
The range of applicability of different theories depends on the water depth, the wave length and the wave height $H$. The boundary between cnoidal and Stokes wave theories is given by an Ursell number (Ur) of 40 (Hedges 1995), where Ur is the ratio between a measure of nonlinearity $(H / d)$, and a measure of shallowness $\left(d^{2} / \lambda^{2}\right)$. The theoretical limit for the highest wave possible, based on the model of Williams (1981), determines the maximum achievable wave height.

Finally, by means of Rienecker-Fenton wave's theory (Fenton 1990), it is possible to achieve a numerical solution to the nonlinear potential problem, without any assumption on the wave depth or height. Indeed, Sobey et al. (1987) shows how well RF waves embrace Stokes' and cnoidal wave theory, in their respective regions of validity. The drawback is that, while there are analytical formulations for calculating the Stokes' and cnoidal waves parameters, the RF approach requires a numerical optimization routine for each wave condition.

\subsubsection{Linear Airy's wave theory}

Assuming a small wave steepness $(s=H / \lambda)$, it is possible to linearize the kinematic and the dynamic free surface boundary conditions, shown in (3b) and (4), respectively, around the SWL, namely at $z=0$. The solution to the linearized potential problem consists of a harmonic free surface elevation and an exponential dynamic pressure, as follows:

$\eta=\frac{H}{2} \cos (k x-\omega t)$,

$p=p_{\mathrm{st}}+p_{\mathrm{dy}}=-\rho g z+\rho g \eta \frac{\cos h k(z+d)}{\cos h k d}$,

where $p_{\mathrm{st}}$ and $p_{\mathrm{dy}}$ are the static and dynamic pressures, respectively, $k=2 \pi / \lambda$ is the wave number, $\omega=2 \pi / T$ is the wave frequency, and $T$ the wave period. Note that, at infinite water depth conditions, the dynamic pressure tends to $\rho g \eta \mathrm{e}^{k z}$.

Pros and cons of Airy's theory are discussed hereafter. Airy's theory is mathematically rigorous and consistent with the velocity potential theory framework, shown in (1)-(4), as long as the linear assumption of small steepness, compared to unity, is verified. Therefore, Airy's theory gives an accurate representation of the wave physics as long as the wave steepness is small. However, in order to compute nonlinear Froude-Krylov forces, pressure specification is required up to the (eventually large positive) free surface elevation, hence introducing modelling errors.

For example, the total pressure $p$ at the free surface $(z=$ $\eta$ ), according to the boundary condition (4), should match the atmospheric pressure, therefore equal to zero. Assuming, for the sake of argument and simplicity, infinite water depth 
conditions, it results that, according to Airy's wave theory, $p$ at the free surface is not zero:

$p=p_{\mathrm{st}}+p_{\mathrm{dy}}=-\rho g \eta+\rho g \eta \mathrm{e}^{k \eta}=\rho g \eta\left(\mathrm{e}^{k \eta}-1\right)$.

As a consequence of the linearization of the free surface boundary conditions, the total pressure is zero at the SWL $(z=0)$ instead, implying a modelling error of the pressure at the actual free surface $(z=\eta)$. Equation (6) shows that such an error is proportional to $\eta\left(\mathrm{e}^{k \eta}-1\right)$, which tends to zero as the wave steepness tends to zero. Similar conclusion can be drawn in generic water depth conditions.

Moreover, such an error depends on the wave number as well. For regular waves, there is only one $k$, which is usually small. Conversely, panchromatic waves are characterized by several frequency components. High-frequency components, with consequently large wave numbers, are particularly problematic for the calculations of the dynamic pressure at positive $z$, since the error increases with the exponential of $k \eta$. Such an issue is further investigated in Sect. 4 .

Finally, note that $p_{\mathrm{st}}$ is defined in the same way for all methods, so it is not a source of error. Consequently, only $p_{\text {dy }}$ will be considered hereafter, when pressure errors are considered. Furthermore, for brevity, the word "pressure" will implicitly refer to $p_{\mathrm{dy}}$, unless differently specified.

\subsubsection{Wheeler's stretching method}

Wheeler's stretching approach (Wheeler 1970) consists of starting from the results obtained with Airy's theory, and apply a convenient change of coordinates, in order to correct the free surface linearized dynamic boundary condition error. Therefore, in the Wheeler's stretching approach, the total pressure is still computed in a linear way, effectively neglecting the nonlinear term. The vertical coordinate $z$ is substituted with $z^{\prime}$, defined as:

$z^{\prime}=d \frac{z+d}{\eta+d}-d$.

Note that the origin of the stretching is located at the sea bottom, since $z^{\prime}=z$ at $z=-d$. On the other hand, $z^{\prime}=0$ at $z=\eta$, hence the dynamic free surface boundary condition is satisfied, and the-linearized-total pressure at the free surface is zero. Moreover, the issue at high-frequency components for panchromatic waves, described in Sect. 2.1.1, is resolved. Notwithstanding that the-linearized-dynamic pressure is correct at $z=\eta$, the whole pressure profile is, in general, an approximation, since it is based on a linear stretching of the results from Airy's theory. Indeed, Wheeler's stretching performs a redistribution of the whole potential field in the vertical direction causing, for example, the Laplace condition (2) to be unsatisfied in the fluid domain.
Therefore, both Wheeler's stretching and Airy's theory are approximations. However, the advantage of Wheeler's stretching over Airy's theory is to describe more realistically the pressure for $z>0$, as shown, for example, by Du et al. (2009) and Clauss et al. (2009). Nevertheless, to the best of the authors' knowledge, there is no previous study considering how the choice of either Airy's theory or Wheeler's stretching affects the calculations of nonlinear Froude-Krylov forces for different wave energy devices: only the region of fluid where the device operates is relevant, as supposed to the whole pressure profile, from sea bottom to the free surface. Consequently, Wheeler's stretching is likely to be particularly effective for floating devices, which operate close to the free surface (where Wheeler's stretching is significantly more accurate than Airy's theory). Further details and discussions are given in Sects. 3.2 and 4.2.

\subsubsection{Rienecker-Fenton wave theory}

The basis of the Rienecker-Fenton method is to write the analytical solution for $\varphi$ in a separated variables form:

$\varphi=\sqrt{\frac{g}{k^{3}}} \sum_{j=1}^{N_{B}} B_{j} \frac{\cos h j k(z+d)}{\cos h j k d} \sin j k x$,

where $B_{j}$ are dimensionless constants for a particular wave, and $N_{B}$ is a finite integer which, according to Fenton (1990), should be chosen between 10 and 20. In order to achieve the same level of accuracy, less steep waves would require less Fourier terms, therefore lower $N_{B}$. In this study, the highest number of components $\left(N_{B}=20\right)$ suggested by Fenton (1990) has been chosen, which is able to accurately describe the steepest waves (Fenton 1990). The truncation of the series for finite $N_{B}$ is the only approximation in this formulation. The values of $B_{j}$ are found numerically, using Newton's method (Fenton 1990).

Note that the complexity of the pressure formulation in the RF method is considerably higher than in Airy's theory and Wheeler's stretching method. In fact, once the $N_{B}$ terms of the potential are obtained, the pressure is computed from Bernoulli's equation (4), which requires the computation of derivatives of the potential. On the other hand, since no approximation is introduced into the formulation of the boundary conditions, the wave pressure profile obtained with the RF method is taken as an accuracy benchmark, and used to evaluate Airy's and Wheeler's approaches. Likewise, the RF approach provides an accurate description of the nonlinear free surface elevation, with higher and steeper peaks compared to linear waves. Figure 2 shows an example of the free surface elevation, using Airy's theory and the RF model. 


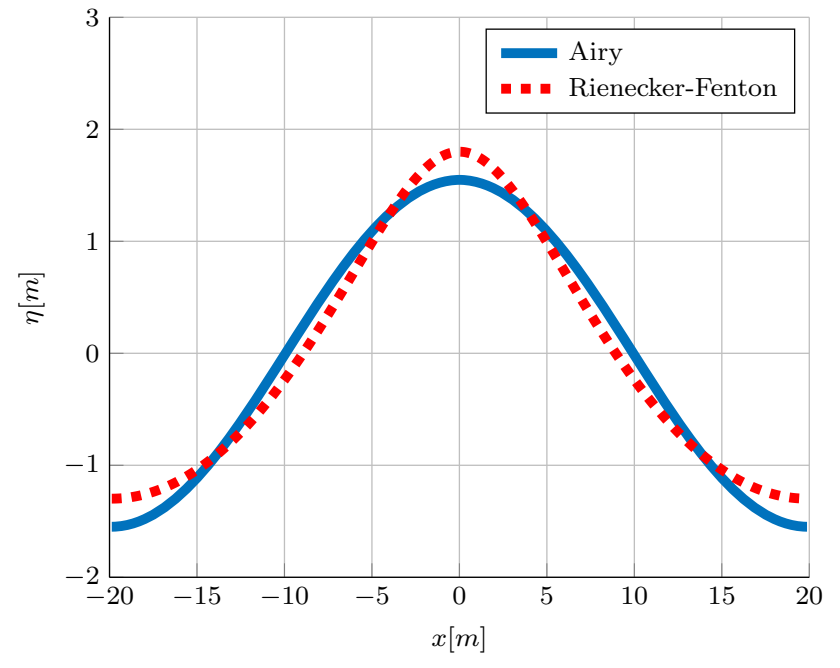

Fig. 2 Free surface elevation for a regular wave condition with $T=5 \mathrm{~s}$, $H=3 \mathrm{~m}, d=12 \mathrm{~m}$

\subsection{Irregular waves}

Irregular sea states are commonly described as a superposition of harmonic components, with frequency-dependent amplitudes and random phases (Ochi 2005). Consequently, assuming that linear superposition is valid, Airy's theory can be used to define the resulting-linearized—wave pressure field:

$p_{\mathrm{dy}}=\sum_{i=1}^{N_{\omega}} \rho g A_{i} \cos \left(k_{i} x-\omega_{i} t+\Phi_{i}\right) \frac{\cos h k_{i}(z+d)}{\cos h k_{i} d}$,

where $N_{\omega}$ is the number of frequency components, $A_{i}, \omega_{i}$, and $\Phi_{i}$ are the amplitude, frequency and phase parameters, respectively, and $k_{i}=2 \pi / \lambda_{i}$ is the wave number.

As in the regular case, for each frequency component, Airy's theory tends to overestimate the pressure at positive displacements (above the SWL), breaking the free surface boundary conditions; such an error is proportional to the exponential of $k \eta$, as shown in (6) and (9). While, in the regular case, wave numbers are relatively small, in the irregular case there are quite large $k_{i}$, associated with high-frequency components of the wave spectrum. The -linearized- dynamic pressure depends linearly on the wave amplitude, and exponentially on the wave number; therefore, in case of large $k_{i}$ and positive $z$, the exponential may dominate on the small amplitude $A_{i}$, which are typically associated with highfrequency components. Consequently, due to the exponential growth of the error, large inaccuracies are expected, close to the free surface, for large wave heights. Note that such an extreme magnification of pressure errors is present only when nonlinear FK forces are considered. Conversely, linear FK force calculation is mathematically consistent with Airy's theory approach, since the pressure integral is com- puted only up to the SWL $(z=0)$, avoiding the exponential growth in pressure error for positive $z$.

Since errors due to the free surface boundary conditions are especially relevant to the irregular case, Wheeler's stretching approach is likely to be particularly effective, since it is designed to verify the linearized free surface dynamic boundary condition at $z=\eta$. As in the regular case, the change of variable, defined in (7), is applied to the pressure formulation in (9). On the other hand, the pressure decay rate, from the free surface to the sea bed, is just an approximation, since it is a simple stretch of Airy's decay. In order to have the correct pressure profile, the full nonlinear boundary problem must be solved.

Furthermore, both Airy's theory and Wheeler's stretching assume linear superposition, implying the absence of energy exchange between different frequency components. As a result, the free surface elevation is a Gaussian process, meaning that the probability distribution of the water displacement is symmetric (Ochi 2005) (peaks and troughs, statistically, are of equal width and height). The Gaussianity of a sea state is influenced only by the water depth: the interaction between frequency components is negligible at infinite water depths, more precisely at significant wave heights $\left(H_{\mathrm{s}}\right)$ such that $d / H_{\mathrm{s}} \geq 4.17$ (Ochi 2005). On the contrary, in intermediate/shallow water depths, higher and narrower peaks, and smaller and larger troughs, may be found.

Non-Gaussian processes can be described through the solution of the nonlinear wave propagation problem (Ducrozet et al. 2016). The higher order spectral method is an efficient approach to the solution of both the nonlinear boundary problem and the nonlinear wave propagation problem. In this work, the open-source HOS-Ocean software is used (Ducrozet 2017). Section 2.2.1 discusses the main features of the method, while full details are given in Ducrozet et al. (2016).

\subsubsection{Higher order spectral method}

The HOS scheme is able to iteratively solve for the nonlinear boundary conditions and the nonlinear sea state evolution, taking into account the energy exchange between different frequency components of the free surface spectrum. HOS models have been widely used and validated, modelling nonlinear energy transfer (Tanaka 2001), or freak waves, (Ducrozet et al. 2007; Sergeeva and Slunyaev 2013), among others.

An efficient solution to the nonlinear potential problem is based on the pseudo-spectral formalism, according to which the velocity potential is expressed through a spectral basis $\Psi_{m}$ : 


$$
\begin{aligned}
\varphi(x, z, t) & =\sum_{m=1}^{N} A_{m}(t) \Psi_{m}(x, z) \\
& =\sum_{m=1}^{N} A_{m}(t) \frac{\cosh k_{m}(z+d)}{\cosh k_{m} d} \mathrm{e}^{i k_{m} x}
\end{aligned}
$$

where $k_{m}=m 2 \pi / L_{x}$ are the wave numbers, and $L_{x}$ is the length of the domain. The HOS scheme is based on the knowledge of the surface quantities $\eta$ and $\widetilde{\varphi}=\varphi(x, \eta, t)$, which are used to evaluate the vertical velocity at the free surface, $W(x, t)$. The method relies on a Taylor series expansion around $z=0$, up to the so-called HOS order $M$ :

$$
\begin{aligned}
& \varphi(x, z, t)=\sum_{m=1}^{M} \varphi^{(m)}(x, z, t) \\
& \varphi^{(1)}(x, 0, t)=\widetilde{\varphi}(x, t) \\
& \varphi^{(m)}(x, 0, t)=-\sum_{k=1}^{m-1} \frac{\eta^{k}}{k !} \frac{\partial^{k} \varphi^{m-k}}{\partial z^{k}}(x, 0, t) \text { for } m>1 .
\end{aligned}
$$

Equations (11) define a triangular system, which is solved iteratively. The solution is then advanced in time, using a fourth-order Runge-Kutta Cash-Carp scheme (Ducrozet et al. 2016), with an adaptive step size. The kinematics and the fluid pressure within the fluid domain are computed via post-processing from free surface quantities, using the modal coefficients $A_{m}$ in (10). Further details about the HOS scheme and its implementation are available in Ducrozet et al. (2016). Ducrozet et al. (2016) proposes as well a validation of the HOS model for regular waves, effectively comparing HOS results with RF theory.

\section{Regular wave results}

The simplest case of steady (regular) waves is considered first. In Sect. 3.1, the accuracy of the pressure profile representation is evaluated for a comprehensive set of wave conditions, from shallow to infinite water depth, and from linear to highly nonlinear waves. Then, in Sect. 3.2, two particular devices are chosen, an HPA and an OWSC, as well as a scatter diagram for a particular installation site, in order to evaluate the impact that the pressure representation accuracy has on the calculation of nonlinear FK forces.

\subsection{Pressure profile}

The overall objective of the study reported in this paper is to evaluate the influence of nonlinear waves on nonlinear FK forces for wave energy converters. Usually, nonlinear models are formulated in the time domain, so that nonlinearities in the equation of motion can be solved in the time progression scheme. Therefore, a time domain framework for the WEC model is assumed hereafter. The two elements affecting the response of the device are the free surface elevation and the pressure profile. On the one hand, $\eta$ is just an input to the system; therefore, its complexity does not affect the complexity of the WEC model, since $\eta$ is evaluated only once. On the other hand, the pressure profile has to be evaluated, and integrated, over the wetted surface of the device, at each time step; therefore, the computational burden of the calculation of nonlinear FK forces is strongly dependent on the complexity of the pressure formulation, though independent of $\eta$. Hence, hereafter, a nonlinear $\eta$ is considered for all wave models. Note that using the undisturbed incident free surface elevation for the calculation of nonlinear FK forces is valid only under the assumption that diffraction and radiation effects are negligible.

The shape of the pressure profile is evaluated for a comprehensive range of wave conditions. Three wave periods, of 5,10 , and $15 \mathrm{~s}$, are considered, and the corresponding wavelengths are computed, according to the water wave dispersion relation (Dean and Dalrymple 1991). Three normalized water depths $d / \lambda$, of $0.05,0.3$ and 0.6 , are considered, respectively, defining shallow, intermediate and infinite water depth conditions (Dean and Dalrymple 1991). Finally, for each wave condition, five wave heights are studied, corresponding to $5,10,20,40$, and $60 \%$ of the theoretical maximum wave height, as defined in Williams (1981). The resulting waves conditions are shown by the ' $x$ ' markers in Fig. 1.

It is worth highlighting that the wave period has an effect of just scaling the free surface elevation and pressure profiles, by changing the wave length; therefore, the markers in Fig. 1 overlap for different values of $T$. Likewise, the normalized pressure profiles for different $T$, but identical $\lambda / d$ and $H / d$, overlap.

Figure 3 shows two representative samples of pressure profiles, at the wave peak $(z=\eta)$, having a wave height equal to $40 \%$ of the theoretical maximum wave height. In infinite water depth conditions, the RF pressure profile significantly differs from Airy's profile only at the free surface, converging to almost the same value at the sea bottom, where the dynamic pressure is almost zero, due to the large distance between the wave and the sea bed. Conversely, large errors are found in shallow water conditions, throughout the entire water depth, since the nonlinear influence of the nearby sea bed is significantly changing the pressure decay rate from the free surface to the bottom.

Wheeler's stretching approach, as explained in Sect. 2.1.2, analytically imposes the correct -linearized- boundary condition at the free surface, and linearly stretches the pressure profile from the sea bottom. Indeed, Fig. 3 shows that the pressure, according to Wheeler's stretching model, is the 

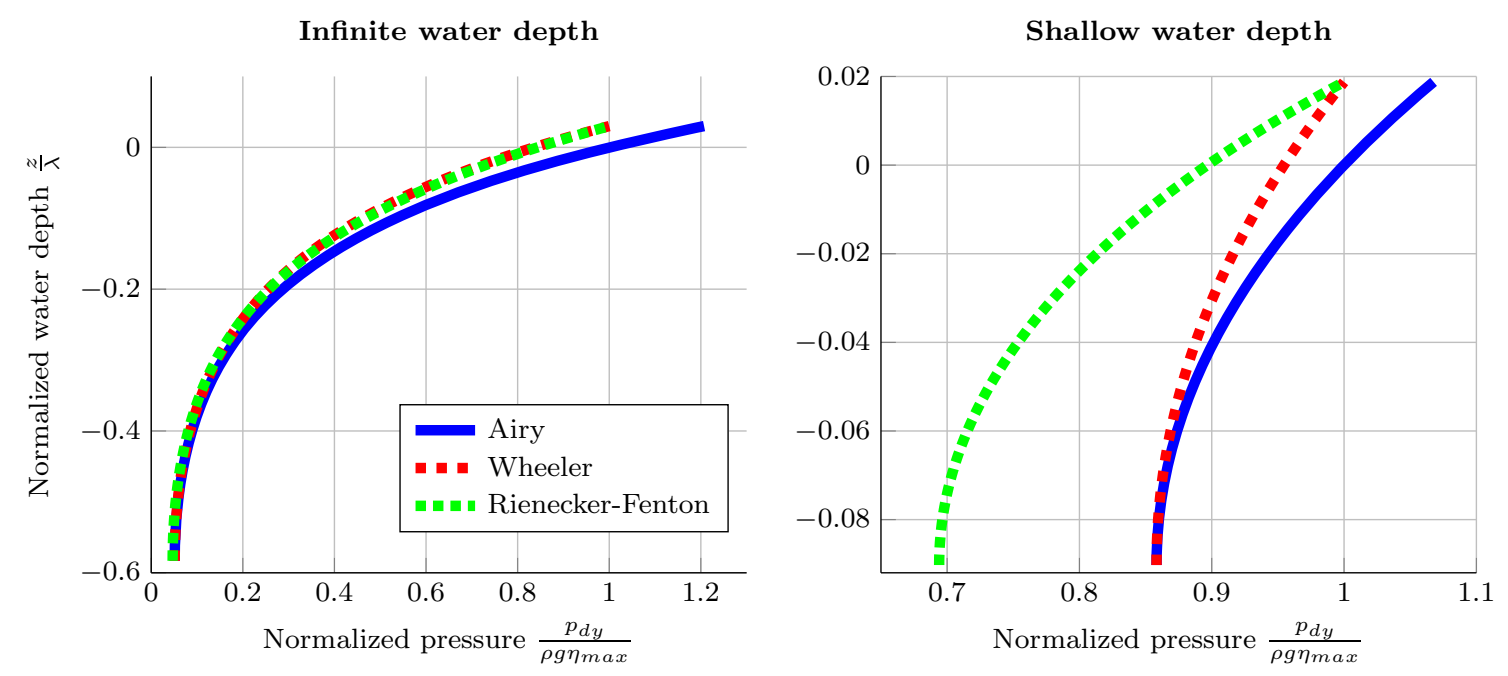

Fig. 3 Normalized pressure profiles for infinite and shallow water depth conditions, for a wave sample with a wave height equal to $40 \%$ of the theoretical maximum wave height

same as the RF one at the free surface, whereas is the same as Airy's one at the sea bottom. Consequently, errors in pressure profile modelling for Wheeler's stretching approach are dependent on the pressure error at the sea bottom, which increases with the decreasing water depth. Finally, note that the pressure is normalized by $\rho g \eta_{\max }$. Consequently, as expected, Airy's theory presents a normalized pressure equal to unity at the SWL. Likewise, Wheeler's stretching and Rienecker-Fenton models show a unity normalized pressure at the actual free surface.

In order to have a more global picture of the errors associated with Airy's theory and Wheeler's stretching model, compared to the RF benchmark model, the mean relative error is computed for all the wave conditions shown by the ' $\mathrm{x}$ ' markers in Fig. 1 as follows:

$\frac{1}{N} \sum_{n=1}^{N} \frac{p_{\mathrm{dy}}^{j}\left(z_{n}\right)-p_{\mathrm{dy}}^{\mathrm{RF}}\left(z_{n}\right)}{p_{\mathrm{dy}}^{\mathrm{RF}}\left(z_{n}\right)}$,

where $p_{\mathrm{dy}}^{j}\left(z_{n}\right)$ is the dynamical pressure computed at point $z_{n}$, using model $j$ (either Airy or Wheeler's stretching). The relative error is evaluated at $N$ vertical points, from the sea bottom to the peak free surface. Given the variety of water depths considered, and trying to define a depth-independent error index, the same number of points is taken for all the waves. On the other hand, since the pressure decay close to the free surface is faster than in deeper water, with a characteristic exponential decay, the $N$ points are chosen with a logarithmic spacing, so that points are denser close to the free surface, and more widely spread towards the sea bed. Figure 4 plots such relative errors against the wave steepness, which is a representative parameter of the degree of nonlinearity in the wave, as discussed in Sect. 2. Finally, note that

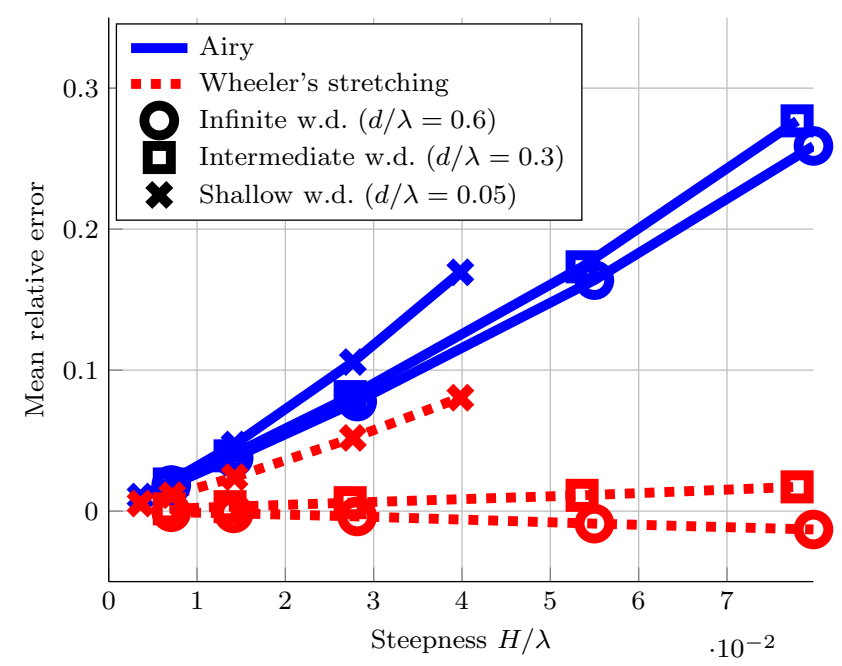

Fig. 4 Relative errors due to Airy's theory and Wheeler's stretching model, compared to the RF benchmark model, for the three water depth (w.d.) conditions. A positive error represents an overestimation of the pressure

a positive relative error represents an overestimation of the pressure.

In general, Fig. 4 shows that the relative error is always increasing with the wave steepness, associated with the amount of nonlinearity in the wave. Nevertheless, for linear waves (steepness lower than $1 \%$ ), the relative error is lower than $2 \%$ for both models, in all depth conditions. On the other hand, as the steepness increases, the curves diverges and the relative error obtained using Airy's theory reaches a maximum of $28 \%$, while with Wheeler's stretching approach, the maximum error is less than $2 \%$ in intermediate/infinite water depths, and about $8 \%$ in shallow water conditions. It 
is evident that Wheeler's stretching model always outperforms Airy's theory; as expected, significant improvements are found at intermediate and infinite water depth conditions, where relative errors are between 12 and 23 times smaller while, at shallow water depth, the relative error is about half.

\subsection{Pressure integral}

The errors, shown in Fig. 4, give an overall evaluation of the pressure profile representation, from the sea bottom to the free surface, according to Airy's and Wheeler's stretching approaches. Nevertheless, as far as nonlinear modelling of wave energy converters is concerned, what is important is the accuracy just in the region of fluid where the device operates, which affects the accuracy of the computation of the nonlinear Froude-Krylov force.

Consequently, the device operating principle has to be taken into account. On the one hand, piercing heaving point absorbers work in the proximity of the free surface, in either infinite or intermediate water depth conditions. On the other hand, oscillating wave surge converters operate in intermediate/shallow water conditions, spanning almost all the depth from the free surface to the sea bed. Therefore, based on Figs. 3 and 4, it can be expected that Wheeler's stretching approach can be more effective for HPAs than for OWSCs, since Wheeler's stretching errors are particularly small close to the surface, and in infinite or intermediate water conditions.

Inspired by the Wavestar (2016) device, a spherical HPA is chosen, with a radius of $2.5 \mathrm{~m}$, and centre at the still water level. The dynamic nonlinear FK force is the integral of the dynamic pressure over the instantaneous wetted surface, which depends on the relative displacement between the device and the free surface elevation. The details for an algebraic resolution of such an integral are given in Giorgi and Ringwood (2017b). The geometry of the OWSC is based on the Oyster 2 device (Aquamarine 2016), where the dynamic FK torque is the resulting torque due to the pressure on the front and rear surfaces of the flap, as shown in Giorgi and Ringwood (2017a).

The reason for considering the device geometry is to quantify the importance of the nonlinear pressure profile in the respective water depths, where the devices operate. Therefore, with the purpose of temporarily excluding geometric nonlinearities, a zero relative displacement is used for the HPA, and the vertical (rest) position is considered for the OWSC. The dynamic pressure is consequently integrated over half a sphere for the HPA, and over a vertical rectangle of $9 \mathrm{~m}$ draft, $3 \mathrm{~m}$ freeboard, and $26 \mathrm{~m}$ width for the OWSC. The freeboard is taken into account for the evaluation of the wetted surface.

Figure 4 shows how modelling errors significantly increase with the wave steepness. Nevertheless, the occurrence of

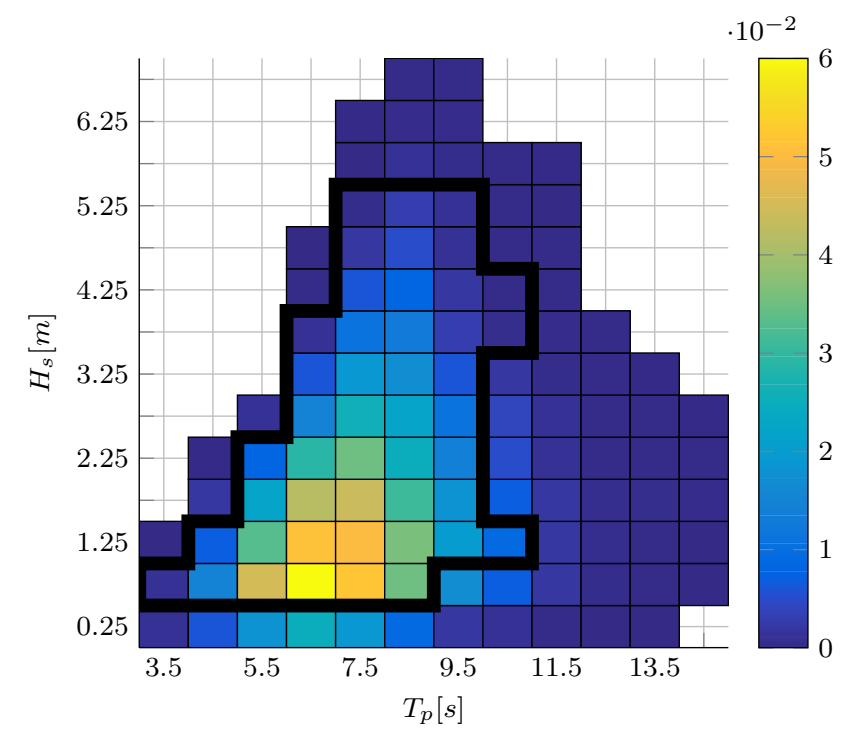

Fig. 5 Significant wave height $H_{\mathrm{s}}$, peak wave period $T_{\mathrm{p}}$ scatter diagram at the European Marine Energy Centre (EMEC), Orkney, Scotland. The solid line defines the operational region of the OWSC Oyster800, which covers $86.1 \%$ of the total wave occurrences (O'Boyle et al. 2015)

highly nonlinear waves is likely to be low, depending on the scatter diagram of the installation site. Furthermore, WEC devices are designed to be operative only in a determined range of wave conditions, where physical constraints are respected. Otherwise, for certain wave conditions, the device is not able to produce energy (if the waves are not energetic enough), or survivability mode is engaged (if the waves are too energetic).

Therefore, for power production assessment studies, the accuracy of the wave representation is important only in the power production region. On the contrary, highly nonlinear and energetic sea states must be considered when the maximum structural loads need to be assessed. Nevertheless, more complex fully nonlinear models, like computational fluid dynamics (CFD), are likely to be necessary for such survivability studies and are beyond the scope of this study.

The scatter diagram considered in this study is shown in Fig. 5, which refers to the European Marine Energy Centre (EMEC), Orkney, Scotland (O'Boyle et al. 2015), where the nominal water depth is $50 \mathrm{~m}$. The solid line defines the operational region of the OWSC Oyster 800, which covers $86.1 \%$ of the total wave occurrences.

A set of regular wave conditions is based on the significant wave heights $\left(H_{\mathrm{s}}\right)$ and peak wave periods $\left(T_{\mathrm{p}}\right)$ shown in Fig. 5. The water depth choice, equal to $13 \mathrm{~m}$, is constrained by the OWSC geometry (Aquamarine 2016). Nevertheless, the HPA is studied in infinite water depth conditions as well. Varying the water depth condition implies changes in the power content of the scatter diagram, since the transition from deep to shallow water happens with non-negligible power 


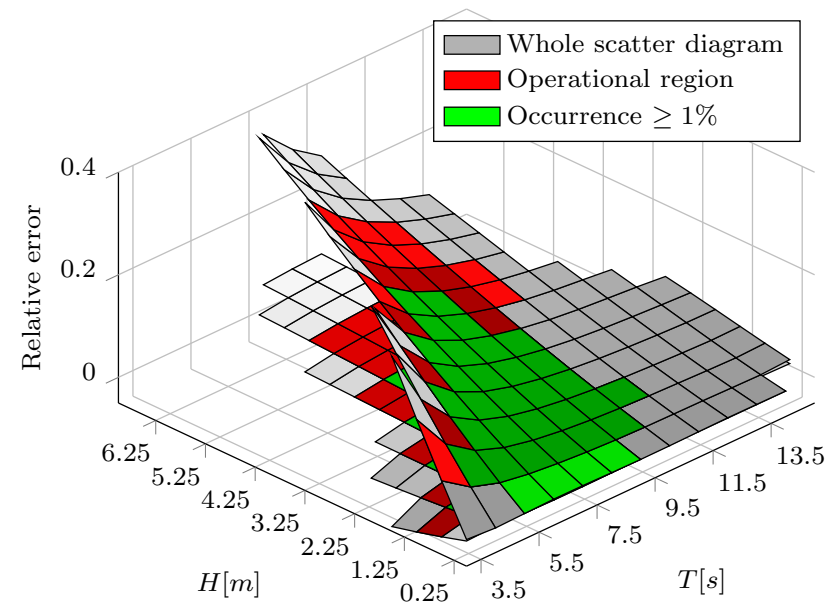

Fig. 6 Relative error in the pressure integral for the HPA (water depth of $13 \mathrm{~m}$ ), using Airy's pressure (on the top), and Wheeler's stretching pressure (on the bottom)

losses. However, in this study, the scatter diagram has been retained unchanged, since the only purpose of considering different water depth conditions is to discuss how the pressure profile changes, due to the proximity (or not) of the sea bottom.

Integrals of the dynamic pressure over the surface of the HPA and the OWSC are computed at the peak of the free surface elevation, in order to highlight nonlinear effects. The computation of such integrals is straightforward for Airy's pressure profile, since algebraic solutions are available, in Giorgi and Ringwood (2017b) for the HPA, and in Giorgi and Ringwood (2017a) for the OWSC. The same algebraic solution can be easily adapted to Wheeler's stretching pressure profile, since just a change of variable is required. On the contrary, integrating the RF pressure profile is much more complex; given the high number of terms in the RF pressure expression, an algebraic solution does not exist . A trapezoidal numerical integration scheme is then used, iterating the integration until an absolute tolerance lower than $1 \mathrm{e}-10$ is achieved..

As in Sect. 3.2, results obtained using the RF model are used as benchmarks to evaluate Airy's and Wheeler's stretching models, whose relative errors are shown in Figs. 6 and 7, for the HPA and the OWSC, respectively. In both figures, the uppermost surface corresponds to Airy's model, whereas the lower surface corresponds to Wheeler's stretching approach, whose errors are always smaller.

Each surface in Figs. 6 and 7 presents three different shades (grey, red, and green). The outer (grey) surfaces cover the whole scatter diagram. The inner darker (red) shades consider only the wave conditions within the operational region of the scatter diagram, shown in Fig. 5, which is the region of interest for power production assessment studies. The inner lighter (green) shades take into account only the wave condi-

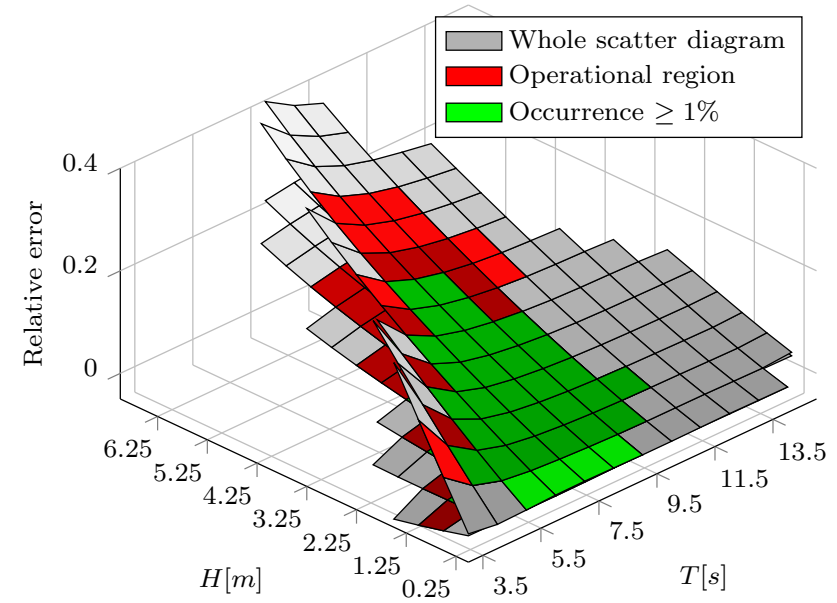

Fig. 7 Relative error in the pressure integral for the OWSC, using Airy's pressure (on the top), and Wheeler's stretching pressure (on the bottom)

tions which pass a significant occurrence threshold, arbitrary set at $1 \%$. Indeed, higher accuracy is required in more probable wave conditions, while larger errors can be accepted for rare wave conditions. The sum of the occurrences over $1 \%$ amount to $87 \%$, which is comparable to $86.1 \%$ of the whole operational region.

Airy's theory produces similar errors in both the HPA and the OWSC, up to $36 \%$ in the full scatter diagram, up to $28 \%$ in the operational region, and up to $18 \%$ in the occurrence threshold region. Conversely, Wheeler's stretching method performs better for the HPA than the OWSC, with maximum errors of 5 and $20 \%$, respectively. Indeed, HPAs work close to the free surface: in this region of fluid, the -linearized- boundary condition error is zero, thanks to Wheeler's stretching change of coordinates.

Finally, the HPA is studied in an infinite water condition as well. Smaller errors are found, compared to the $13 \mathrm{~m}$ water depth condition. Airy's theory maximum error drops from 32 to $25 \%$, while Wheeler's stretching maximum error drops from 5 to $4 \%$. A summary of the maximum errors in each of the three regions (whole scatter diagram, operational region, and occurrence threshold region) is tabulated in Table 1.

\section{Irregular wave results}

As in Sect. 3, the accuracy of the pressure representation is evaluated, for irregular waves, across the whole sea depth, in Sect. 4.1, and in relation to nonlinear FK forces on an HPA and an OWSC, in Sect. 4.2.

All the 108 sea states in the EMEC scatter diagram, shown in Fig. 5, are considered, using JONSWAP spectra with $\gamma=3.3$. Constant water depth, of $13 \mathrm{~m}$, is assumed, as in Sect. 3.2. Note that, even though the HOS software solves the nonlinear wave propagation problem, treating all sea states 
Table 1 Maximum errors for the whole scatter diagram, the operational region, and occurrence threshold region, for the HPA and the OWSC

\begin{tabular}{|c|c|c|c|c|c|c|}
\hline & \multicolumn{2}{|l|}{$\mathrm{HPA}_{\infty}$} & \multicolumn{2}{|l|}{$\mathrm{HPA}_{13}$} & \multicolumn{2}{|l|}{$\mathrm{OWSC}_{13}$} \\
\hline & Airy $(\%)$ & Wheeler (\%) & Airy $(\%)$ & Wheeler $(\%)$ & Airy $(\%)$ & Wheeler (\%) \\
\hline Occurrence $\geq 1 \%($ total $87 \%)$ & 14.1 & -1.0 & 17.3 & 3.1 & 18.2 & 9.1 \\
\hline Operational boundary (total $86.1 \%$ ) & 20.6 & -2.4 & 26.0 & 4.4 & 27.8 & 14.7 \\
\hline Whole scatter diagram (total $100 \%$ ) & 25.1 & -4.2 & 32.5 & 5.3 & 35.8 & 20.5 \\
\hline
\end{tabular}

The subscript indicates the water depth for each of the device

as potentially non-Gaussian, only 41 sea states, out of 108 (38\%), are actually non-Gaussian. Indeed, the remaining 67 sea states can be considered predominantly Gaussian processes, since $d / H_{\mathrm{s}} \geq 4.17$ (Ochi 2005).

Two-dimensional HOS simulations are run with HOSOcean, in a spatial domain 40 times longer than the peak wave length $\lambda_{\mathrm{p}}$ of the sea state, using $N=256$ sampling points (see (10)), as in (Ducrozet et al. 2016). The HOS order $M$, in (11), is chosen equal to 5 , based on a convergence study for the steepest sea state $\left(H_{\mathrm{s}}=4.75 \mathrm{~m}, T_{\mathrm{p}}=6.5 \mathrm{~s}\right)$, which is the most challenging to simulate, where the sea state steepness is defined as $H_{\mathrm{s}} / \lambda_{\mathrm{p}}$. The convergence test is based on the free surface horizontal velocity at the highest wave crest.

Note that, in the most severe sea state considered, there are chances that some waves break, since the ratio $H_{\mathrm{s}} / \lambda_{\mathrm{p}}$ is larger than 0.064 (Ducrozet et al. 2017). In case of wave breaking, the HOS method may not converge to a solution. Ducrozet et al. (2017) give the most important guidelines to set-up the HOS model in order to reduce the risk of failure and, in general, ensure appropriate levels of accuracy. In this paper, since only one set of random phases is studied for each sea state, it happens that, by chance, the HOS does not diverge for the one particular realization considered.

Finally, each simulation is run for 250 times the peak period, in order to guarantee proper nonlinear wave propagation, and to have a representative statistical description of the sea state. The post-processing, useful to extract the pressure profile in the fluid domain, is performed at $x=0$, with a sampling rate of $1.28 \mathrm{~Hz}$.

\subsection{Pressure profile}

In order to apply Airy's and Wheeler's stretching method to compute the pressure field distribution, the frequency components (frequency, amplitude and phase) of the free surface elevation are needed, which can be obtained through a fast Fourier transform (fft). Given the significant length of the time signals $\left(250 T_{\mathrm{p}}\right)$, at a sampling rate of $1.28 \mathrm{~Hz}$, the $\mathrm{fft}$ of the whole signal would require a very large number of components, which would considerably slow down post-processing calculations. Therefore, the time series are divided in 25 segments, of equal length (10 $\left.T_{\mathrm{p}}\right)$, which is considered appropriate to be representative of the sea state. Apart from the practical advantage, the segmentation is useful to obtain some statistical information about the mean error computed for each sea state.

From each segment, a fft is used to calculate an energy spectrum. Note that such time series are not periodic; consequently, the reconstructed time signal (obtained through an inverse $\mathrm{fft}$ of the energy spectrum) overlaps with the original one at all the sampling points, except for the very last point. A fair comparison of the three wave modelling approaches (Airy's theory, Wheeler's stretching, and HOS), must consider the very same surface elevation in all cases; therefore, the last point is discarded.

For each point in time, the pressure profile is compared, along the whole water depth. Figure 8 shows an example of wave profiles for a sea state in the middle of the scatter diagram, with $T_{\mathrm{p}}=8.5 \mathrm{~s}$ and $H_{\mathrm{s}}=3.25 \mathrm{~m}$. Both a wave trough and a wave peak are considered, whose absolute value is chosen to be about half of $H_{\mathrm{s}}$, in order to be representative of the irregular sea state. Note that, similarly to Fig. 3, the normalized pressure for Airy's theory is unity at the still water level $(z=0)$, while for Wheeler's stretching and the HOS, the normalized pressure is unity at the actual free surface $(z=\eta)$.

Some preliminary qualitative considerations are offered about the sign of the error, either positive (overestimation), or negative (underestimation), where the HOS pressure profile is taken as a benchmark. On the one hand, at the free surface $(z=\eta)$, Airy's theory overestimates the pressure for positive $\eta$, but underestimates it for negative $\eta$, due to the approximation of the free surface dynamic boundary condition around the SWL. Note that errors for positive $\eta$ can be particularly large, due to the exponential increasing pressure, and the relatively large wave number of some frequency components, as discussed in Sect. 2.2. On the other hand, Wheeler's stretching approach produces negligible errors at the free surface, which is indeed the objective of the stretching.

At the sea bottom $(z=-d)$, it is not possible, in general, to anticipate the sign of the error. Nevertheless, if the water depth tends to infinity, the dynamic pressure tends to zero, for all three methods. Therefore, the pressure profile, according to Airy's theory, would remain always on the 
A wave trough: $\eta=-1.58 m$

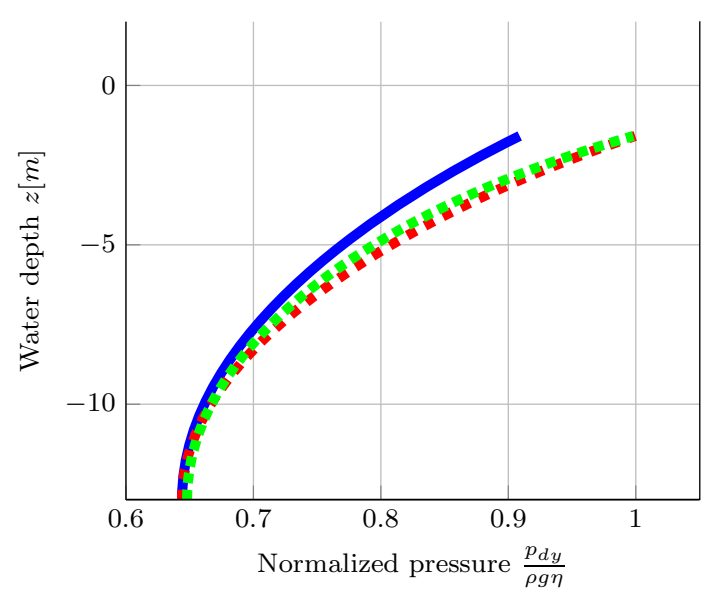

A wave peak: $\eta=1.65 m$

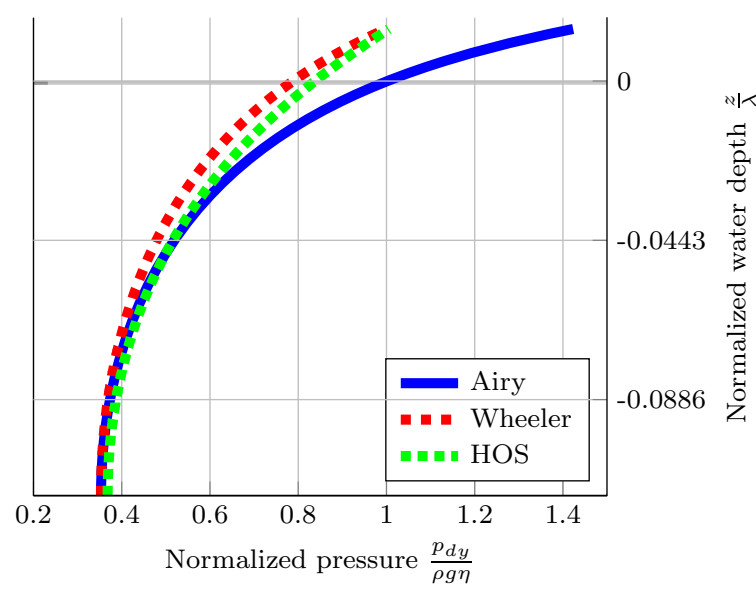

Fig. 8 Normalized pressure profiles for a sea state with $T_{\mathrm{p}}=8.5 \mathrm{~s}$ and $H_{\mathrm{s}}=3.25 \mathrm{~m}$, at $13 \mathrm{~m}$ water depth, taken at a wave trough (on the left) and a wave peak (on the right), whose absolute value is about half of $H_{\mathrm{s}}$

same side of the HOS pressure profile (either over- or underestimating), and the sign of Airy's theory error would remain constant across the entire water depth, converging to zero as the depth increases. However, with finite water depth, the pressure at the sea bottom is not negligible, as shown, for example, in Fig. 3; hence larger errors are expected, either positive or negative. Therefore, the pressure profile, according to Airy's theory, may intersect the HOS profile, causing a change of sign of the error as the depth increases. Likewise for Wheeler's pressure profile, which is just a stretch, centred at the sea bottom, of Airy's pressure profile.

For quantitative considerations, due to the eventual change of sign of the pressure error across the fluid domain, the absolute error is considered instead, in order to avoid errors of opposite sign cancelling out. The information about the sign of the error, which is lost with such an absolute error, is recovered in Sect. 4.2, when the resulting nonlinear FK forces are considered.

The error is normalized by the maximum characteristic pressure of the sea state, defined as $\rho g H_{\mathrm{s}} / 2$. For each point in time, the average of all the errors, at different depths from the sea bottom to the free surface elevation, is considered. Afterwards, for each data segment, the time average is considered:

$\frac{1}{\rho g H_{\mathrm{s}} / 2} \frac{1}{N M} \sum_{m=1}^{M} \sum_{n=1}^{N}\left|p_{\mathrm{dy}}^{j}\left(z_{n}, t_{m}\right)-p_{\mathrm{dy}}^{H O S}\left(z_{n}, t_{m}\right)\right|$,

where $p_{\mathrm{dy}}^{j}\left(z_{n}, t_{m}\right)$ is the dynamic pressure, according to model $j$, computed at $z_{n}$ in space, and $t_{m}$ in time, with $t_{1}=0$ and $t_{M}=10 T_{\mathrm{p}}$. Consequently, for each sea state, there are 25 estimates of the overall error, from which the mean and the $95 \%$ confidence interval (CI) are computed, which are tabulated in Table 2. The bold line represents the operational region, as in the scatter diagram in Fig. 5. The (cyan) shading of the cells is proportional to the steepness of the sea state.

The mean overall errors, in Table 2, are shown in Fig. 9 as well, where the horizontal axis represents a progressive enumeration of the sea states, based on a vertical reading of Table 2: starting from the first column (constant $T_{\mathrm{p}}$ ), the enumeration increases with $H_{\mathrm{s}}$, until the row is full; then, the enumeration continues to the following column. Consequently, in Fig. 9, $T_{\mathrm{p}}$ is constant between two consecutive peaks of the error curves, while $H_{\mathrm{s}}$ increases from left to right. Indeed, as expected, errors increase with increasing values of $H_{\mathrm{s}}$.

From both Table 2 and Fig. 9, it can be noticed that, as expected, Wheeler's stretching always outperforms Airy's theory. Nevertheless, while large improvements are appreciable for severe sea states, smaller differences are found in milder sea conditions. Therefore, it is important to weight the performance of the two approaches with the probability of occurrence of each sea state, and whether or not the sea state is within the power production region. Consequently, similarly to the regular wave case in Sect. 3.2, Fig. 9 shows the occurrence curve, a $1 \%$ occurrence threshold, and the sea states which are outside the operational region of the device (shaded in red). It is evident that larger errors, as expected, occur for sea states which are outside the operational region, and/or are relatively rare. Nevertheless, even though the performance of Airy's theory and Wheeler's stretching is similar in the operational region, Wheeler's stretching is preferred, since it is always more accurate, while maintaining the same (low) complexity of Airy's theory.

An important variable, which is missing from Fig. 9, is the sea state steepness. Indeed, the two sea state parameters, influencing the pressure error, are the sea state steepness and the significant wave height. Figure 10 plots the overall 
Table 2 Mean overall pressure error and 95\% confidence interval (CI), normalized by $\rho g H_{\mathrm{s}} / 2$ and multiplied by $10^{2}$, according to Airy's theory and Wheeler's stretching

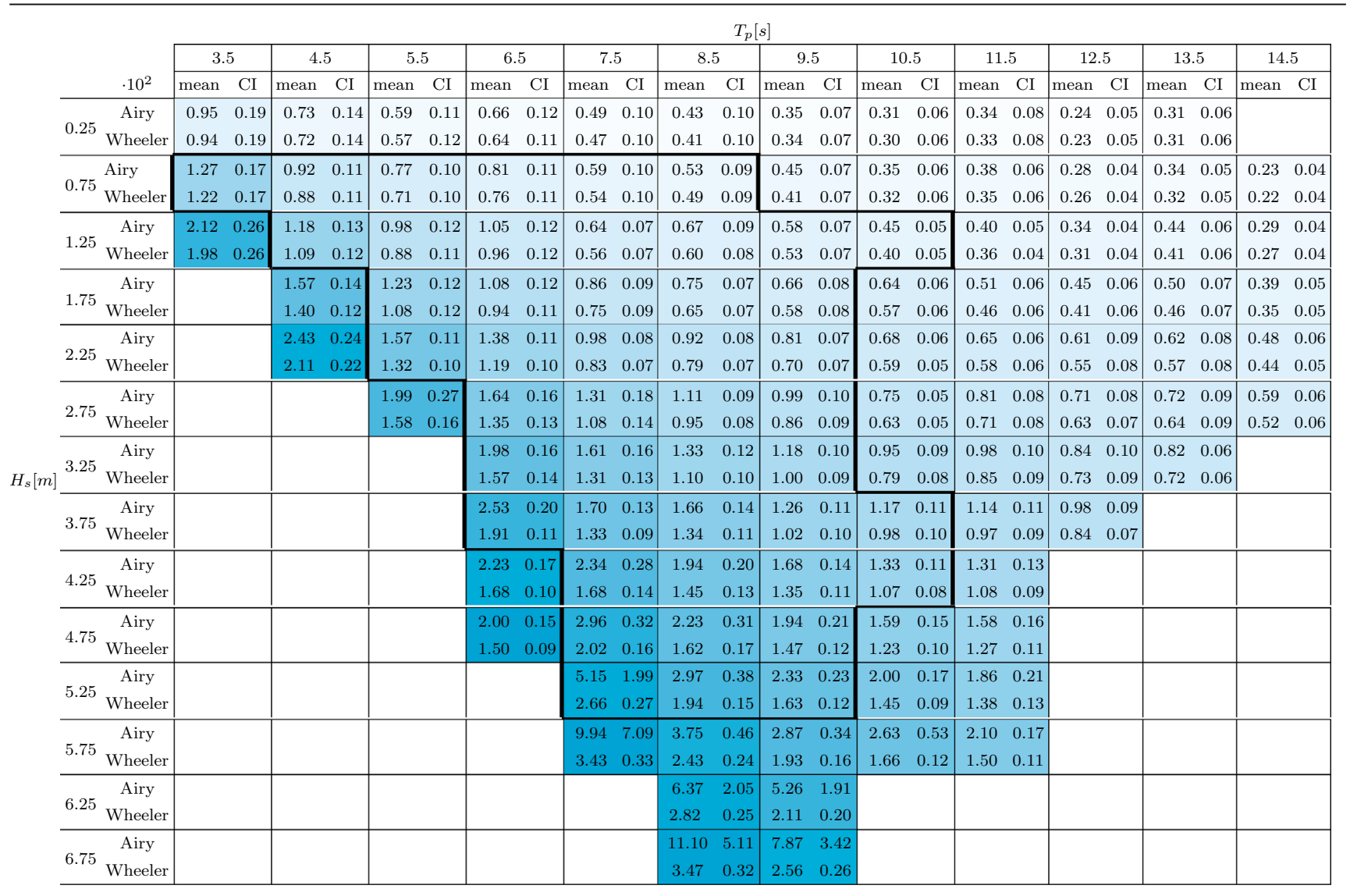

The bold line represents the operational region, as in the scatter diagram in Fig. 5, at $13 \mathrm{~m}$ water depth. The (cyan) shading of the cells is proportional to the steepness of the sea state

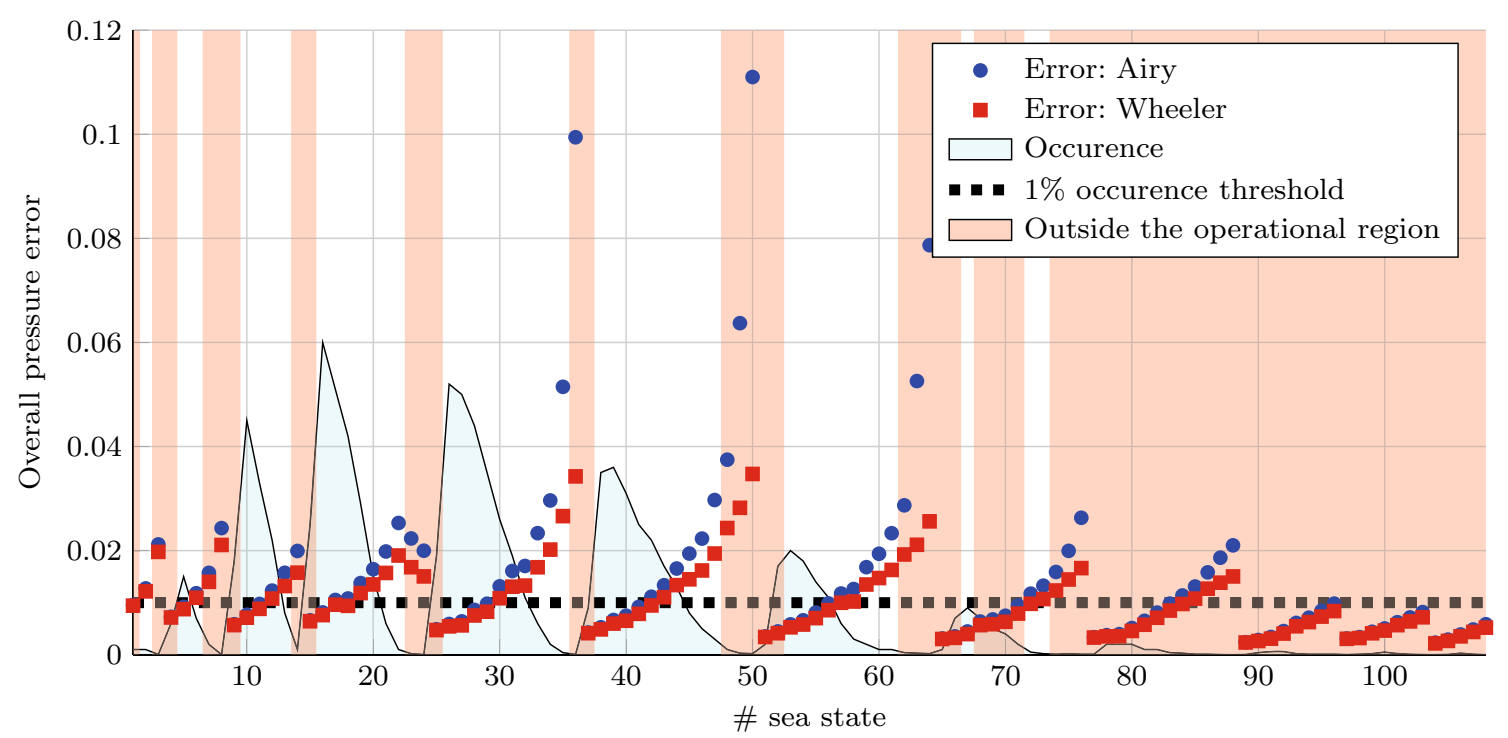

Fig. 9 Overall pressure error, as in Table 2, with a progressive enumeration of the sea states, following the columns of Table 2. The occurrence of each sea state is shown, according to the scatter diagram, in Fig. 5. The sea states outside the operational region are shaded (in red) 


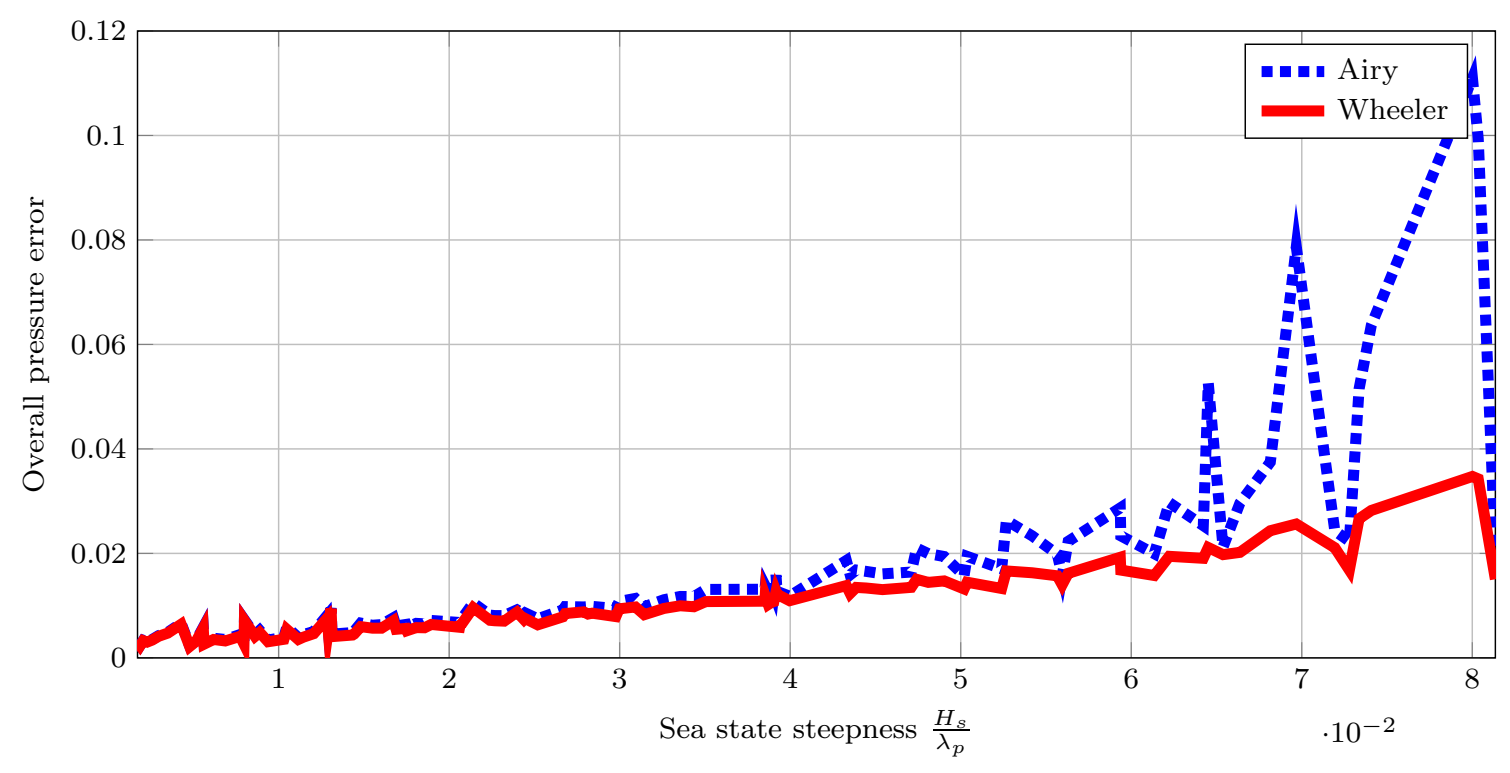

Fig. 10 Overall pressure error, as in Table 2, plotted against the sea state steepness

pressure errors of Table 2 against the sea steepness. Recall that, as explained in Sect. 4, for a wave steepness larger than 0.064 , wave breaking is possible. The general trends clearly show an increasing error with the wave steepness, but there are significant oscillations, especially for Airy's theory, for large steepness values, which are due to the wave height. In fact, a similarly large steepness can be obtained with a short but small sea state (for example $T_{\mathrm{p}}=3.5 \mathrm{~s}$ and $H_{\mathrm{S}}=1.25 \mathrm{~m}$ ), or a long but high sea state (for example $T_{\mathrm{p}}=8.5 \mathrm{~s}$ and $H_{\mathrm{s}}=6.75 \mathrm{~m}$ ), as shown by the shading of the cells in Table 2. Consequently, with almost the same steepness, much larger errors are obtained for larger $H_{\mathrm{s}}$. Such variations are particularly evident in Airy's theory approach, since significant errors are obtained for large waves.

\subsection{Pressure integral}

The impact of the dynamic pressure representation accuracy on the nonlinear FK force calculations is investigated, for a hypothetical HPA and an OWSC installed at EMEC, with a water depth of $13 \mathrm{~m}$. As in Sect. 3.2, the objective is to introduce representative geometries for an HPA and an OWSC, which can highlight the essential differences due to the operating principles. Therefore, on the one hand, the HPA is represented by a half-submerged floating sphere, with $5 \mathrm{~m}$ diameter, i.e. with the centre of gravity at the free surface elevation. On the other hand, the OWSC is represented by a $26 \mathrm{~m}$ wide vertical rectangle, with $9 \mathrm{~m}$ draft and $3 \mathrm{~m}$ freeboard, which is taken into account for the evaluation of the wetted surface.
Consequently, the HPA is strongly affected by the large errors that Airy's theory produces close to high crests, due to the high-frequency components of the spectrum. Conversely, the OWSC is constrained by its limited freeboard; when large waves occur, the OWSC becomes fully submerged, and hence avoids the free surface dynamic boundary condition errors of Airy's theory.

A single estimate of the model accuracy, across the whole time series, would be useful. Since the nonlinear FK force is oscillating, between positive and negative values, the definition of a fair relative error, for each time step, would be challenging, due to the changes of sign, and divisions by quasi-zero values. Furthermore, an average over the whole time series should be taken. Rather, the area $\Gamma$ under the absolute value of the nonlinear FK force $\left(F_{\mathrm{FK}}\right)$ time series, is considered instead:

$\Gamma=\int_{0}^{250 T_{\mathrm{p}}}\left|F_{\mathrm{FK}}\right| \mathrm{d} t$

$\Gamma$ has the advantage of being a single, positive value, characterizing the whole time series, and conveying an overall under/overestimation information. An accuracy index is then defined as the ratio between Airy's theory (or Wheeler's stretching) area, and the HOS area. Figure 11 shows such an accuracy index, for the HPA and the OWSC, using Airy's theory and Wheeler's stretching, highlighting which sea states are outside the power production region.

Consistent with Figs. 9 and 10, Wheeler's stretching performs better than Airy's theory, showing smaller deviations from the unity accuracy index. Furthermore, Wheeler's 


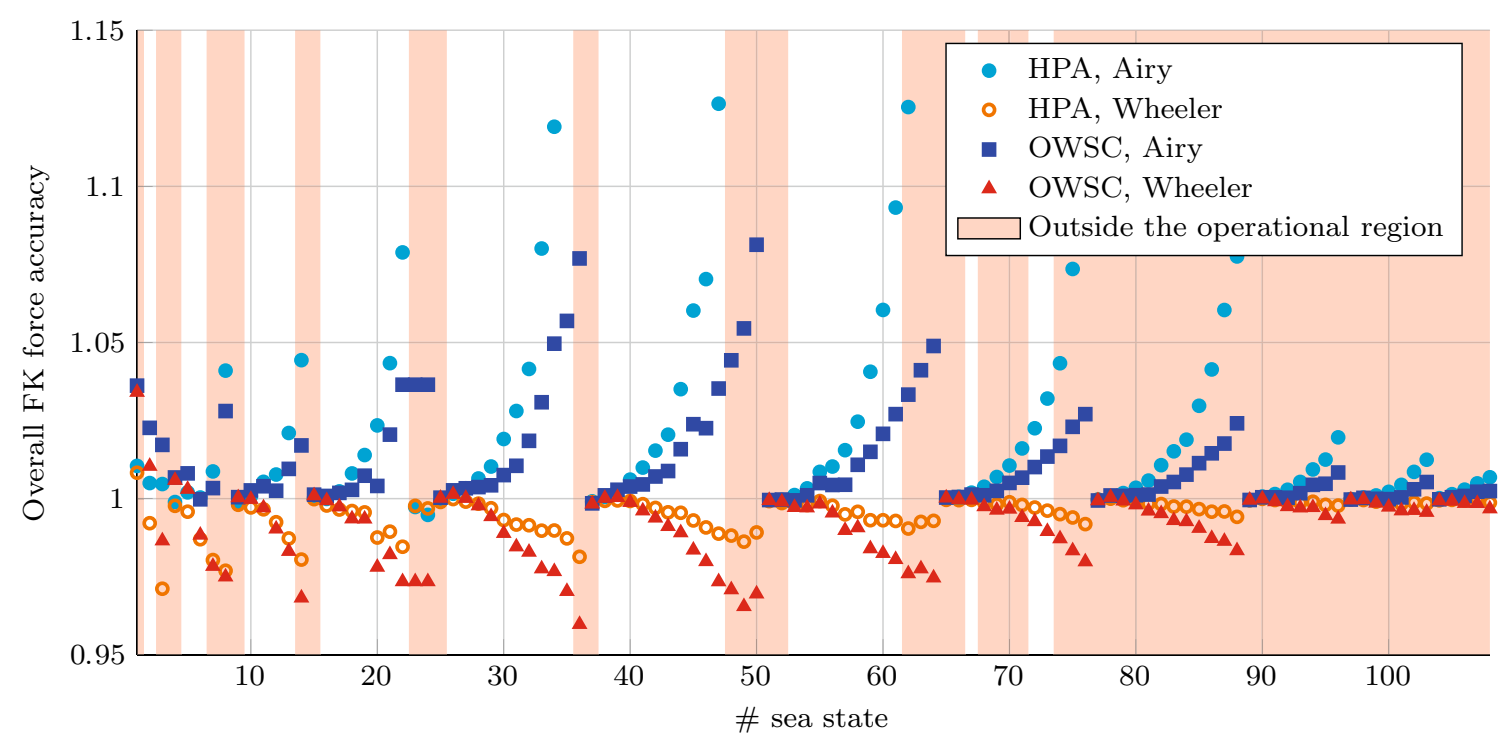

Fig. 11 Froude-Krylov accuracy index, defined as the ratio between the areas under the absolute value of the nonlinear FK time series, where indices greater than unity represent overestimations of the FK force

stretching overall underestimates the nonlinear FK force, whereas Airy's theory overestimates it.

Despite the fact that the two devices are subject to the same pressure errors, the essential geometric differences, between the HPA and the OWSC, cause significant variations of the FK force accuracy. Indeed, Airy's theory performs worse for the HPA, in particular for quite extreme sea states (errors up to 2.01). Note that, in order to improve the readability of Fig. 11, the upper limit of the vertical axis is set to 1.15, excluding HPA Airy's errors for the six most extreme sea states. Nevertheless, such sea states are outside the power production region.

Such results for the HPA with irregular waves contrast with those for regular waves, shown in Figs. 6 and 7. Airy's theory is affected by two errors: the free surface dynamic boundary condition error and the entire pressure profile error. Due to their dimensions and positions in the fluid domain, the HPA is mainly affected by the free surface error, whereas the OWSC by the pressure profile error. In the irregular wave case, the free surface dynamic boundary condition error can become particularly large, due to high-frequency components, as discussed in Sect. 4.1, which are absent in the regular wave case.

Conversely, since Wheeler's stretching resolves the dynamic boundary condition error, it is affected only by the pressure profile error. Therefore, consistent with the results for regular waves, Wheeler's stretching is more accurate for the HPA than the OWSC, even with irregular waves, since the HPA is located in a smaller region of fluid, close to the free surface, where Wheeler's representation is most accurate.

\section{Conclusions}

The accuracy of nonlinear hydrodynamic models for wave energy converters is directly influenced by the fidelity of the wave field representation. However, accuracy improvements have to be weighted by the increase in complexity and computational cost, required to implement more accurate wave models. Using regular and irregular waves, this paper considers four different modelling approaches, namely linear Airy's theory, Wheeler's stretching approach, the Rienecker-Fenton method (only for regular waves), and a higher order spectral method (only for irregular waves). In particular, the focus of the study is on the impact of the accuracy of the pressure field representation on nonlinear Froude-Krylov force calculations. Note that nonlinear Froude-Krylov force models rely on the assumption of small radiation and diffraction effects, which is usually the case for devices with small dimension compared to the wave length, or subject to small movements.

The entire pressure profile is evaluated for a comprehensive range of wave conditions, both regular and irregular. Modelling error magnitudes depend on the water depth, wave steepness, and wave height. In all cases, Airy's theory produces larger errors than Wheeler's stretching, especially with irregular sea states, due to the relatively high-frequency components of the wave spectrum.

As far as WEC nonlinear models are concerned, only the region of fluid where the device operates is relevant for the final accuracy of the results. On the one hand, Figs. 6 and 7 show that, when regular waves are considered, smaller errors are produced in HPAs, with respect to OWSCs, for both Airy's theory and Wheeler's stretching. On the other hand, Fig. 11 shows that, with irregular waves, consistent results 
are found only for Wheeler's stretching, which is more accurate for HPAs. Conversely, Airy's theory performs better for OWSCs than HPAs.

Furthermore, it is important to highlight that the performance of the wave models have to be weighted with the occurrence of each sea condition, according to the scatter diagram of the installation site. An further criterion to consider is the power production region, which is the only one taken into account for power production assessment studies.

Finally, it can be concluded that the Wheeler's stretching approach is a convenient wave modelling option for computing nonlinear Froude-Krylov forces for wave energy devices, since it performs well compared to RF/HOS methods, and performs always better than Airy's theory, but maintaining the same level of complexity.

Acknowledgements This paper is based upon work supported by Science Foundation Ireland under Grant No. 13/IA/1886.

\section{References}

Aquamarine (2016) Aquamarine power. http://www.aquamarinepower. $\mathrm{com} /$

Babarit A, Mouslim H, Clément A, Laporte-Weywada P (2009) On the numerical modelling of the non linear behaviour of a wave energy converter. In: ASME 2009 28th international conference on ocean, offshore and arctic engineering. American Society of Mechanical Engineers, pp 1045-1053

Clauss G, Kosleck S, Sprenger F, Boeck F (2009) Adaptive stretching of dynamic pressure distribution in long-and short-crested sea states. In: ASME 2009 28th international conference on ocean, offshore and arctic engineering. American Society of Mechanical Engineers, pp 333-343

Dean RG, Dalrymple RA (1991) Water wave mechanics for engineers and scientists, vol 2. World Scientific Publishing Co Inc, London

Du S, Hudson D, Price W, Temarel P (2009) Implicit expressions of static and incident wave pressures over the instantaneous wetted surface of ships. Proc Inst Mech Eng Part M J Eng Marit Environ 223(3):239-256

Ducrozet G (2017) Open-source release of HOS-ocean. https://github. com/LHEEA/HOS-ocean/wiki

Ducrozet G, Bonnefoy F, Le Touzé D, Ferrant P (2007) 3-d HOS simulations of extreme waves in open seas. Natl Hazards Earth Syst Sci 7(1):109-122. https://doi.org/10.5194/nhess-7-109-2007. http:// www.nat-hazards-earth-syst-sci.net/7/109/2007/

Ducrozet G, Bonnefoy F, Le Touzé D, Ferrant P (2016) HOS-ocean: open-source solver for nonlinear waves in open ocean based on high-order spectral method. Comput Phys Commun 203:245-254

Ducrozet G, Bonnefoy F, Perignon Y (2017) Applicability and limitations of highly non-linear potential flow solvers in the context of water waves. Ocean Eng 142:233-244

Fenton J (1990) Nonlinear wave theories. Sea 9(Part A):3-25

Gilloteaux JC (2007) Mouvements de grande amplitude d'un corps flottant en fluide parfait. application à la récupération de l'énergie des vagues. $\mathrm{PhD}$ thesis, Ecole Centrale de Nantes-ECN
Giorgi G, Ringwood JV (2017a) Comparing nonlinear hydrodynamic forces in heaving point absorbers and oscillating wave surge converters. J Ocean Eng Mar Energy 1-11. https://doi.org/10.1007/ s40722-017-0098-2

Giorgi G, Ringwood JV (2017b) Computationally efficient nonlinear Froude-Krylov force calculations for heaving axisymmetric wave energy point absorbers. J Ocean Eng Mar Energy 3(1):21-33

Giorgi G, Ringwood JV (2017c) Froude-Krylov and viscous drag representations in nonlinear wave energy devices models in the computation/fidelity continuum. Ocean Eng 141:164-175

Giorgi G, Penalba M, Ringwood JV (2016) Nonlinear hydrodynamic models for heaving buoy wave energy converters. In: Proceedings of the 3rd Asian wave and tidal energy conference, pp 144-153

Guerinel M, Jansson E, Todalshaug JH, Jesmani M, Guijt K (2017) Modelling alternatives for a heaving point absorber with and without stiffness modulation. In: 12th European wave and tidal energy conference (EWTEC), Cork

Hedges T (1995) Regions of validity of analytical wave theories. In: ICE proceedings water maritime and energy, vol 112, pp 111-114

Le Méhauté B (1976) An introduction to hydrodynamics and water waves. Ocean Front Coast Process. Springer, Berlin. https://doi. org/10.1007/978-3-642-85567-2

Merigaud A, Gilloteaux JC, Ringwood JV (2012) A nonlinear extension for linear boundary element methods in wave energy device modelling. In: ASME 2012 31st international conference on ocean, offshore and arctic engineering. American Society of Mechanical Engineers, pp 615-621

Newman J (1977) Marine hydrodynamics. MIT Press, Cambridge

O’Boyle L, Doherty K, van't Hoff J, Skelton J (2015) The value of full scale prototype data-testing oyster 800 at emec, orkney. In: Proceedings of the 11th European wave and tidal energy conference (EWTEC), Nantes, France, pp 6-11

Ochi MK (2005) Ocean waves: the stochastic approach, vol 6. Cambridge University Press, Cambridge

Penalba M, Giorgi G, Ringwood JV (2017) Mathematical modelling of wave energy converters: a review of nonlinear approaches. Renew Sustain Energy Rev 78:1188-1207. https://doi.org/10.1016/j.rser. 2016.11.137, http://www.eeng.nuim.ie/coer/wp-content/uploads/ 2017/05/J270MRGG-3.pdf

Sergeeva A, Slunyaev A (2013) Rogue waves, rogue events and extreme wave kinematics in spatio-temporal fields of simulated sea states. Natl Hazards Earth Syst Sci 13(7):17591771. https://doi.org/10.5194/nhess-13-1759-2013. http://www. nat-hazards-earth-syst-sci.net/13/1759/2013/

Sobey RJ, Goodwin P, Thieke RJ, Westberg RJ Jr (1987) Application of Stokes, cnoidal, and Fourier wave theories. J Waterw Port Coast Ocean Eng 113(6):565-587

Tanaka M (2001) Verification of Hasselmann's energy transfer among surface gravity waves by direct numerical simulations of primitive equations. J Fluid Mech 444:199-221. https://doi.org/10.1017/ S0022112001005389

Tarrant KR (2015) Numerical modelling of parametric resonance of a heaving point absorber wave energy converter. $\mathrm{PhD}$ thesis, Department of Mechanical \& Manufacturing Engineering, Trinity College

Wavestar (2016) Wavestar a/s. http://wavestarenergy.com/

Wheeler J (1970) Methods for calculating forces produced on piles in irregular waves. J Petrol Technol 249:359-367

Williams J (1981) Limiting gravity waves in water of finite depth. Philos Trans R Soc Lond A Math Phys Eng Sci 302(1466):139-188 